\title{
REVISITANDO PORT LEUCATE-BARCARÈS
}

\author{
Izol Emilia MAREZ LÓPEZ
}

Este artículo revisa los materiales originales del proyecto Port Leucate-Barcarès, en Languedoc-Roussillon, concebido por Georges Candilis; magnífico ejemplo de las acciones del gobierno francés durante los ańos sesenta para desarrollar el turismo de masas en el litoral mediterráneo. Este estudio se basa en textos e imágenes inéditas del archivo personal y profesional de Georges Candilis.

Mediante un análisis crítico, se pretende conocer los valores, relaciones y aportaciones del Movimiento Moderno a la arquitectura y al urbanismo del turismo de masas, así como conocer las repercusiones e influencias de este proyecto en la concepción del espacio turístico internacional.

\section{Port Leucate-Barcarès, arquitectura turística, Georges Candilis, Movimiento Moderno en arquitectura}

\section{REVISITED PORT LEUCATE-BARCARÈS}

This article reviews the original materials of the Port Leucate-Barcarès project, in Languedoc-Roussillon, conceived by Georges Candilis; a magnificent example of the French government's actions during the sixties to develop mass tourism on the Mediterranean coast. This study relies on unpublished texts and images from Georges Candilis personal and professional files.

Through critical analysis, we expect to know the values, relationships and contributions of the Modern Movement in architecture and urbanism of mass tourism, as well as the repercussions and influences of this project on the conception of international tourist space.

Port Leucate-Barcarès, leisure architecture, Georges Candilis, Modern Movement in Architecture 


\section{Revisitar el pasado para entender el presente}

El debate y la crítica sobre la arquitectura del turismo suelen afrontar la cuestión como si se tratara de un fenómeno urbanístico espontáneo, sin un proyecto arquitectónico y urbano que le preceda, olvidando que algunas de las operaciones más importantes de colonización y desarrollo turístico no solo estuvieron económicamente ordenadas por planes a escala nacional, sino también por un proyecto urbanístico llevado a cabo por profesionales de reconocido prestigio.

El objeto de este artículo, así como el de la tesis leída en diciembre de 2012, es investigar sobre uno de los casos ejemplares desarrollados en Francia, en la década de los sesenta, en la costa del Languedoc-Roussillon, en Port LeucateBarcarès, cuyo proyecto estuvo a cargo del arquitecto Georges Candilis, architecte en chef de esta estación turística y uno de los primeros en reflexionar sobre el papel y la aportación de la arquitectura moderna en este campo.

Para ello, hay que retroceder en el tiempo, inmediatamente después de la Segunda Guerra Mundial, y recordar cuáles fueron los postulados y políticas de vivienda que se llevaron a cabo en este período.

En aquellos momentos, el gobierno francés tuvo que afrontar la reconstrucción del país y el déficit de vivienda. Apostó por los principios formulados por el Movimiento Moderno y por la utilización del orden abierto ${ }^{I}$ como la solución arquitectónica y urbanística para solucionar el problema del hábitat. La construcción masiva de viviendas se realizó normalmente en la periferia de los grandes centros urbanos, mediante operaciones que fueron bautizadas muy gráficamente como grands ensembles. La rigidez y falta de flexibilidad de los principios establecidos en la Carta de Atenas y su mala aplicación originó un movimiento crítico de jóvenes arquitectos, la tercera generación del Movimiento Moderno, agrupados en torno al llamado TEAM 10, que plantearon la necesidad de reformular las bases del movimiento a partir de principios más urbanos.

En Francia, el equipo formado por los arquitectos Candilis-Josic-Woods fue el principal divulgador de las teorías de este grupo y su exponente más representativo de la construcción de vivienda masiva (Fig. 1).

1 El arquitecto francés Jacques Lucan en su libro Architecture en France (1940-2000) denomina orden abierto (ordre ouverte) al conjunto de principios urbanos producto de la Carta de Atenas. El concepto ordre ouverte no es utilizado comúnmente en textos traducidos al castellano pero en este estudio se ha traducido literalmente. 

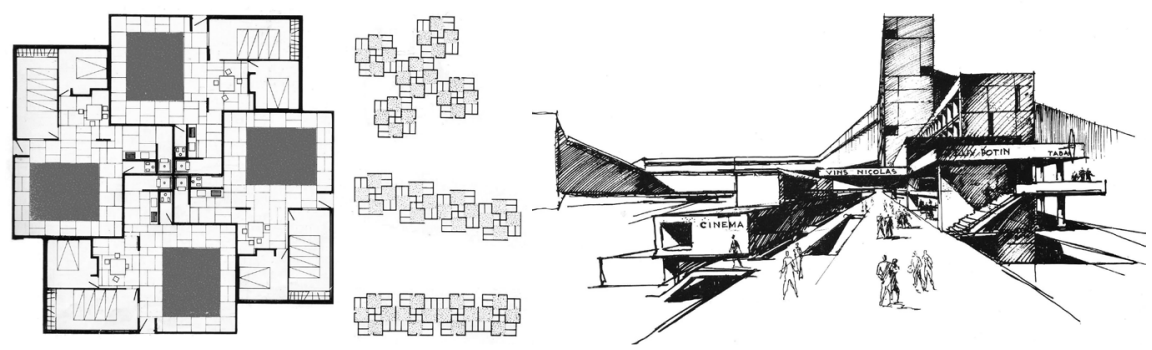

Fig.1: Grupo de cuatro viviendas articuladas alrededor de instalaciones sanitarias. Fuente: CANDILIS, Georges; JOSIC, Alexis; WOODS, Shadrach. Une décennie d'architecture et d'urbanisme. Paris: Eyrolles, 1968.

Fig 2: Estudio de calle-centro lineal. Fuente: CANDILIS, Georges; JOSIC, Alexis; WOODS, Shadrach. Toulouse-Le Mirail. La naissance d'une ville nouvelle. Stturgart: Kart Krämer Verlag, 1975.

Estos autores intentaron resolver la cuestión del espacio colectivo, que tan poco había interesado a los fundadores del Movimiento Moderno, a través de la definición de elementos urbanos como la calle-lineal (stem) (Fig. 2), normalmente acompañada de megaestructuras verticales y horizontales (web) que debía actuar de centro urbano, así como repensar la vivienda a partir de las experiencias que se venían desarrollando en la propia ciudad. Sus formulaciones tuvieron un eco especial a partir de dos grandes proyectos en los que intervinieron: Toulouse-Le Mirail y la Universidad Libre de Berlín, en los que pusieron en práctica algunos de estos principios.

El debate que interesa desarrollar en este artículo afecta a los trabajos y reflexiones llevados a cabo por Georges Candilis cuando se disolvió el equipo inicial y él asume la dirección de la transformación turística del LanguedocRousillon. A partir de ello, Candilis construye uno de los primeros discursos teóricos sobre la arquitectura del ocio, en base a sus experiencias en las colonias francesas y sus propuestas para la ordenación de aquella costa.

\section{Ordenación turística del litoral de Languedoc-Roussillon}

En la década de 1960 las acciones que se llevaron a cabo para afrontar el desarrollo urbanístico de la costa mediterránea francesa fueron consecuencia de la necesidad de reactivar económicamente algunos de los territorios más deprimidos del país, dedicados a la agricultura, a través de la actividad turística. Uno de estos proyectos implementados por el gobierno francés fue la Ordenación turistica del litoral de Languedoc-Roussillon a cargo de la Délégation à l'aménagement du territoire et à l'action régionale (DATAR). Para ello se creó una Misión Interministerial (Operación Racine) ${ }^{2}$, encargada de llevar a cabo la política turística determinada por la DATAR.

2 El director de esta Misión fue un alto funcionario de gobierno de nombre Pierre Racine. Por ello, a veces se nombra la Comisión con el apellido de quien la presidía 


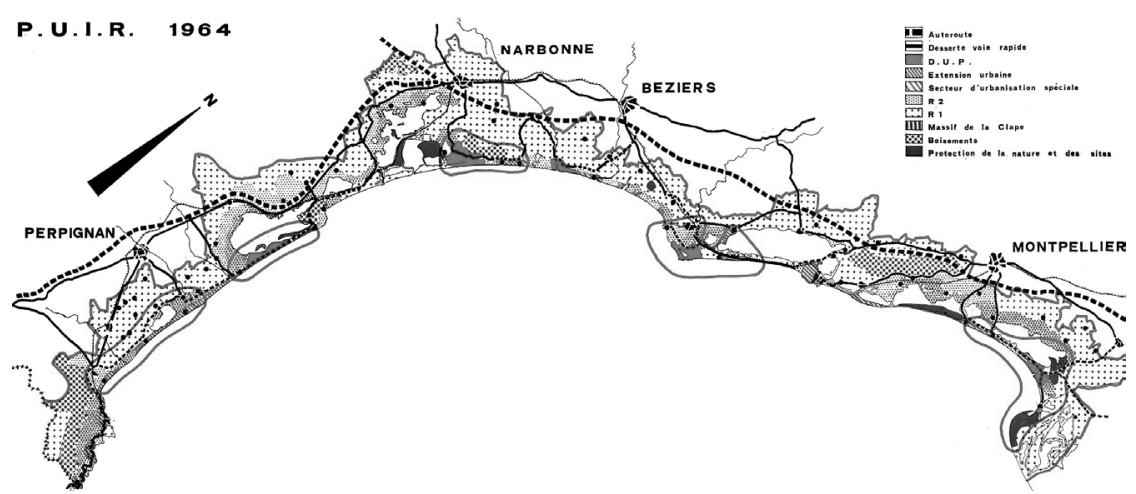

Fig 3: Plan de Urbanismo de Interés Regional de 1964. Fuente: Fondo CANGE. Dossier 373 IFA 104/1. Centre d'Archives d'architecture du XXe siècle de l'Institut français d'architecture.

En 1964 se aprobó el Plan de urbanismo de interés regional (PUIR), el cual propuso una ordenación del litoral basada en una secuencia de unidades turísticas separadas por grandes espacios verdes, estaciones turísticas ${ }^{3}$ ex-nihilo ${ }^{4}$ y un sistema de ports de plaisance, que incluía puertos existentes y de nueva creación (figura 3).

En 1963, antes de la presentación oficial del plan, para adecuar la región a las necesidades del desarrollo turístico, se realizaron algunas acciones como la erradicación de los mosquitos y la adquisición adelantada de terrenos para evitar la especulación.

Los proyectos de las nuevas estaciones turísticas fueron realizados por un grupo de arquitectos, representantes de aquella tercera generación de la que se ha hablado, dirigido por Candilis. Todos los proyectos plantearon un nuevo modelo de ciudad balnearia en el que se eliminaba el paseo marítimo y se tomaba el port de plaisance como el elemento concentrador de las principales actividades de la estación. Las primeras estaciones construidas fueron las dos situadas en los extremos de la región: La Grande-Motte y Port Leucate-Barcarès.

\section{Port Leucate- Barcarès}

Localizada cerca de Perpinán, la estación turística Port Leucate-Barcarès pertenece a la unidad turística del mismo nombre, la cual incluye el sitio

3 Traducción del francés de la denominada station touristique, manera común para referirse a una ciudad que dispone de equipamiento turístico importante, favorece las estancias temporales y las actividades de ocio. También denominadas con menor frecuencia ville touristique.

4 La condición ex nihilo convirtió a las estaciones turísticas de Languedoc-Roussillon en las primeras villes nouvelles modernas promovidas en los años sesenta del siglo pasado por el Estado Francés a través de la DATAR, antecediéndose casi una década a las villes nouvelles promovidas también por la DATAR para controlar la excesiva concentración urbana de la región parisina. 


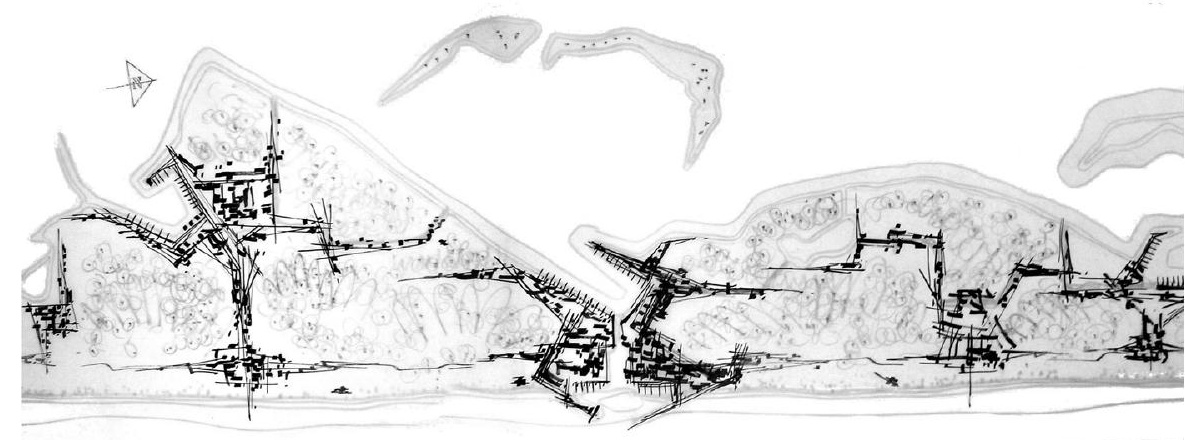

Fig.4: Esquema preliminar Port Leucate-Barcarès (1963). Fuente: Fondo CANGE. Dossier 236 IFA 50/3. Centre d'Archives d'Architecture du XXe siècle de l'Institut français d'architecture.

natural que une físicamente a las estaciones turísticas Leucate y Barcarès. El terreno tiene una longitud aproximada de $8 \mathrm{~km}$ que colinda al Este por el mar mediterráneo y al Oeste por la laguna de nombre Étang de Salses-Leucate.

El architecte en chef responsable de la concepción y construcción de Port Leucate-Barcarès fue Georges Candilis, quién en esta ocasión no trabajó con sus compañeros Alexis Josic y Shadrach Woods. Candilis estuvo al frente de un cabinet rodeado de arquitectos jóvenes durante casi 15 años, hasta 1977, cuando fue sustituido por el arquitecto Michel Duplay.

\section{a) Anteproyecto urbano}

En el Centre d'Archives d'architecture du XXe siècle de l'Institut français d'architecture existen documentos inéditos que registraron el proceso creativo de Georges Candilis en el proyecto Port Leucate-Barcarès. El anteproyecto es lo que mejor muestra la adaptación de los conceptos teóricos formulados junto con Alexis Josic y Shadrach Woods, inmersos los tres en la etapa de revisión de los principios modernos de la Carta de Atenas, formando parte del grupo de arquitectos del TEAM 10.

En un primer anteproyecto de Georges Candilis, el terreno no observa importantes transformaciones y mantiene su estado natural. A manera de esbozo se observa una megaestructura horizontal (web) que recorre todo el sitio en forma zigzagueante, compuesta por núcleos urbanos que concentran actividades en edificaciones de diversos tamaños, densidades y niveles. Los núcleos urbanos están unidos entre sí mediante ramificación urbanas menos densas. Un canal divide las dos estaciones y conecta el mar con la laguna. El port de plaisance principal está localizado en dicho canal, con infraestructura tanto en el mar como en la laguna. Dos puertos menores orientados hacia la laguna están situados en ambos extremos del conjunto (figura 4). 


\section{b) Proyecto urbano}

El anteproyecto de Port Leucate-Barcarès sufrió un cambio radical respecto al proyecto final, que no conservó la solución de grandes estructuras concentradoras de actividades y viviendas que recorren todo el terreno. Fue decantado por una propuesta urbana basada en un sistema jerárquico de vialidades que penetran la estación y diseminaron en todo el terreno los asentamientos urbanos.

El proyecto urbano de Port Leucate-Barcarès está basado en los principios urbanos del orden abierto: zonificación, jerarquía vial con privilegio al peatón y el emplazamiento de los edificios en grandes áreas verdes. Además, se integró el concepto stem, utilizado por el equipo Candilis-Josic-Woods en sus conjuntos de viviendas, en una modalidad de menor escala denominada rue commercial, que une al peatón con el comercio y al comercio con la vivienda.

Fig.6: Zonificación de Port Leucate-Barcarès. Fuente: Propia. Fig.7: Sistema de Movilidad de la estación Port Leucate-Barcarès. Fuente: Propia.

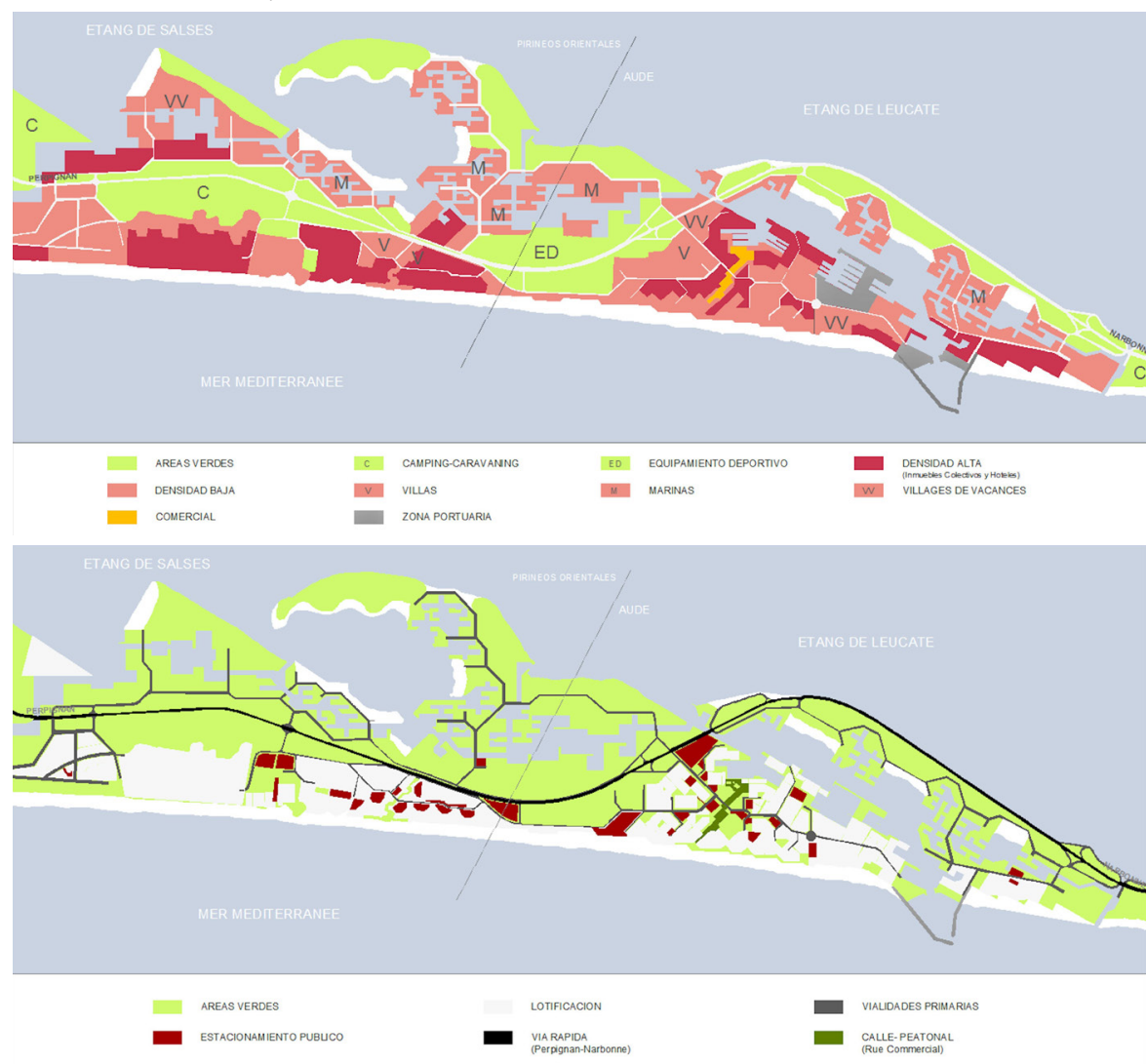



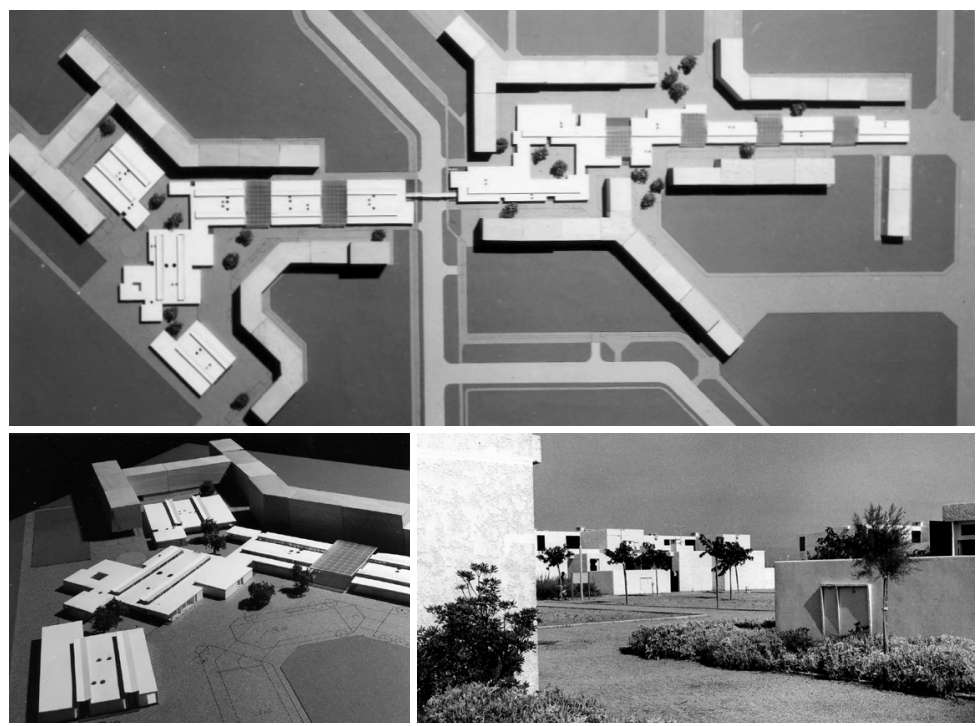

Fig.10: Maqueta de rue commercial con pasillo cubierto. Fuente: Fondo CANGE-G-67-3. Dossier 236 IFA 431/1. Centre d'Archives d'Architecture du XXe siècle de l'Institut français d'architecture. Fig.11: Jardín comunitario de un conjunto de vivienda individual. Fuente: Fondo CANGE-G. Centre d'Archives d'Architecture du XXe siècle de l'Institut français d'architecture.

mar, fue concebido rompiendo el esquema tradicional: es peatonal, permite el acceso directo a la playa sin obstáculo alguno y concentra equipamientos efímeros de playa (pista ciclista, área de juegos, plazas, kioscos, puesto de socorro y área de baños) (figura 9).

El equipamiento urbano y turístico forma una red que penetra toda la estación en diferentes escalas y fue agrupado en puntos estratégicos para estimular la animación. A escala de la estación, además de las instalaciones portuarias, se propuso un complejo deportivo y recreativo que incluyó estadio y piscina olímpica, ubicados al centro del conjunto.

A escala de barrio, se proyectaron calles peatonales denominadas rue commercial para concentrar los principales comercios y establecimientos administrativos cercanos a las viviendas (figura 10). La red se complementó con equipamiento turístico situado de manera puntual: canchas de tenis, club hípico, piscinas, restaurantes, hoteles, salas de cines, módulos sanitarios, vestidores, un museo de esculturas en la arena y el paquebote Lydia en la arena utilizado como restaurante y discoteca.

El sistema de espacios libres está compuesto por áreas verdes públicas y privadas, plazas de estacionamiento público y espacio público destinado a deportes, recreación y descanso. El área verde privada es el área no construida dentro de los conjuntos de vivienda individual o villages de vacances, que de 


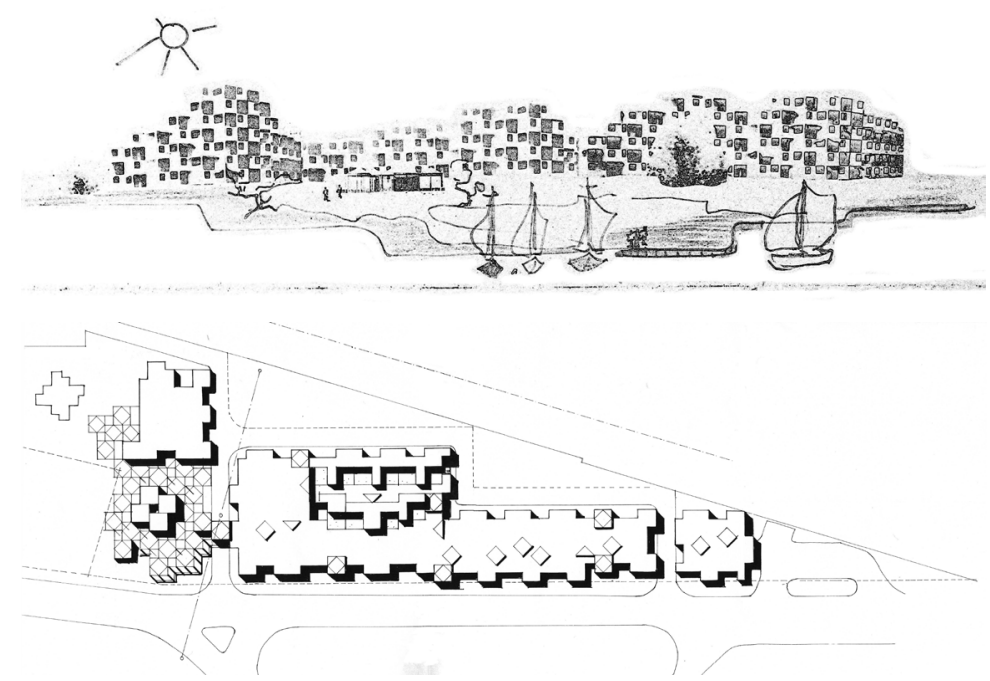

Fig.12: Estudio de viviendas de vacaciones. 1960-1973. Fuente: Fondo CANGE-G-60. Dossier 236 IFA 646/3. Centre d'Archives d'Architecture du XXe siècle de I'Institut français d'architecture. Fig.13: Megaestructura comercial. Fuente: Fondo CANGE-G. Centre d'Archives d'Architecture du XXe siècle de I'Institut français d'architecture.

acuerdo con el reglamento deberían ser destinados a jardines comunitarios (figura 11). El área verde pública flanquea la vía primaria; se encuentra también en el boulevard náutico para dar al visitante la impresión de que se llega a un lugar en contacto directo con el paisaje natural.

\section{c) Arquitectura}

Georges Candilis afirma que una nueva actividad social como el turismo exigía una nueva arquitectura en armonía con la naturaleza del lugar. En Port Leucate-Barcarès planteó una arquitectura con un espíritu que respondiera a las exigencias de la vida mediterránea y al ocio: "Un problème nouveau exige une architecture nouvelle. La diversité des sites et des activités exige également une diversité architecturale, pour que sépanouisse un nouveau milieu humain, en harmonie avec de merveilleux éléments offerts par la nature: montagne, vallée, forêt, mer, soleil, neige, eau...".

El fondo de archivos de Georges Candilis del Centre d'Archives d'Architecture $d u X X e$ siècle de l'Institut français d'architecture conserva un estudio de viviendas de vacaciones que sintetiza la propuesta arquitectónica de Candilis

5 "Un problema nuevo exige una arquitectura nueva. La diversidad de sitios y de actividades exigen igualmente una diversidad arquitectónica para que se desarrolle un nuevo medio humano, en armonía con los maravillosos elementos ofrecidos por la naturaleza: montaña, valle, bosque, mar, sol, nieve, agua...". CANDILIS, GEORGES. "Analyse critique de la place des loisirs dans l'architecture et l'urbanisme contemporains" en L'Architecture d'Aujourd'hui, núm. 131, avril-mai, 1967. 

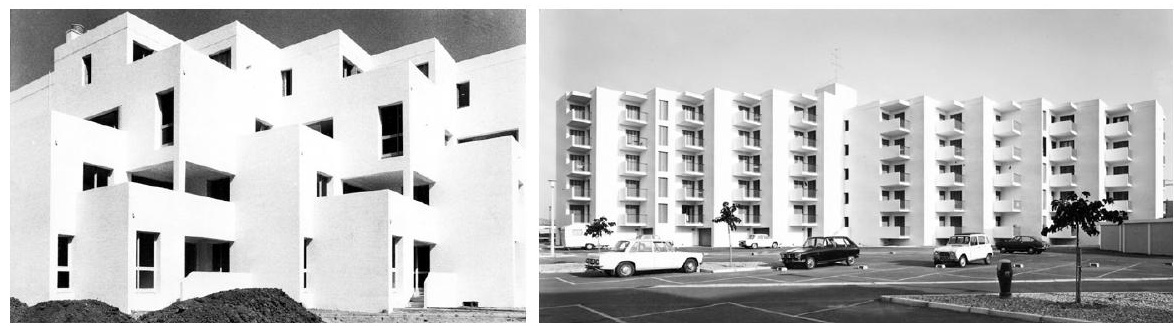

Fig.14: Inmueble colectivo con terrazas escalonadas. Fuente: Fondo CANGE-G. Centre d'Archives d'Architecture du XXe siècle de l'Institut français d'architecture. Fig.15: Acoplamiento a $45^{\circ}$ de Inmueble colectivo. Fuente: Fondo CANGE-G. Centre d'Archives d'Architecture du XXe siècle de l'Institut français d'architecture.

para una arquitectura del ocio. En el dibujo hecho a mano, al frente, se observa un muelle con una serie de barcos encallados. Al fondo, un conjunto de inmuebles escalonados forman casi un panal de abeja que entrelaza terrazas y habitaciones con vista al mar. En el medio, se ubica equipamiento de playa con una arquitectura sencilla y diáfana. El estudio demuestra la adaptación e integración de la arquitectura moderna a las actividades náuticas (figura 12).

Para la arquitectura del equipamiento urbano fueron adaptadas megaestructuras horizontales y tramas reticulares integradas en escala y forma a la vivienda, evitando contrastes urbanos y monumentalidad. Todo lo contrario: Candilis recurrió al concepto de antimonumentalidad para crear una ciudad de vacaciones que borrara en el usuario la idea de la ciudad dedicada al trabajo, en la cual las construcciones en altura rompen con el paisaje. Pabellones prefabricados para uso recreativo, comercial y náutico fueron utilizados siguiendo indistintamente las mismas especificaciones modulares y estructurales (figura 13).

Con la intención de ofrecer un turismo para todos, la estación incluyó tipologías innovadoras y alternativas que responden a las necesidades de una nueva sociedad. La diversidad tipológica de hábitat del ocio y una mezcla de densidades amplió posibilidades de elección adecuadas a las preferencias y medios económicos de los visitantes; en general, la arquitectura para el alojamiento fue sencilla y económica, buscando estar al alcance de todos.

Georges Candilis clasificó el hábitat en acoplamientos horizontales y verticales. Los acoplamientos horizontales se caracterizan por que cada lote está ocupado por una sola vivienda, pero existe una articulación con las otras viviendas. Los acoplamientos verticales responden a la necesidad de asegurar una densificación puntual, obtener el máximo número de viviendas con vista al mar y crear una relación directa entre las instalaciones turísticas y viviendas.

Todos los acoplamientos presentan una trama principal para la ordenación del conjunto y una secundaria para cada vivienda. La integración de la vivienda a una trama de conjunto la hace formar parte de un tejido urbano 
o le permite formar un nuevo tejido urbano. La utilización de estas tramas facilitó organizar las agrupaciones, asegurar una continuidad estructural y racionalizar la construcción. Los elementos normalizados y estandarizados utilizados en las viviendas permitieron la variedad de acoplamientos. Algunas viviendas fueron simplemente delimitadas con muros de sustentación al exterior y al interior, con muros de separación de materiales ligeros.

Los inmuebles para alojamientos colectivos fueron destinados a hoteles, moteles y vivienda colectiva. En la propuesta original no existen las torres; por el contrario, se construyeron edificios de cuatro a seis niveles para que no contrastaran con el terreno plano. La tipología propuesta fue el edificio bloque dispuesto de manera individual o agrupada. En ocasiones, aparte de los alojamientos colectivos, los conjuntos integraron viviendas individuales (villas) y equipamiento turístico (piscinas, restaurantes, locales comerciales, áreas de descanso y espacios deportivos).

Los elementos arquitectónicos más sobresalientes de los inmuebles colectivos fueron las terrazas, logias y balcones que dan la posibilidad de tener contacto directo con la naturaleza y disfrutar de la vista del mar y el paisaje. En algunos casos, las terrazas aparecen escalonadas e intercaladas provocando un juego de volúmenes (figura 14). El sistema de acoplamiento a $45^{\circ}$ aumentó las posibilidades de la vista al mar y aseguró la privacidad de las terrazas (figura 15). Los elementos de articulación vertical (escaleras, elevadores, tragaluces) son resaltados en los inmuebles que presentan cambios de dirección en su composición.

La arquitectura de las viviendas colectivas de Port Leucate-Barcarès es muy similar a la de los grands ensembles construidos por el equipo Candilis-Jo-

Fig.16: Célula tipo de villa-patio del village de vacance C. I. L. (1967). Fuente: Fondo CANGE-G-65-3. Centre d'Archives d'Architecture du XXe siècle de l'Institut français d'architecture. Fig.17: Acoplamiento horizontal de villas-patio de una planta. Fuente: Fondo CANGE-G. Centre d'Archives d'Architecture du XXe siècle de I'Institut français d'architecture.
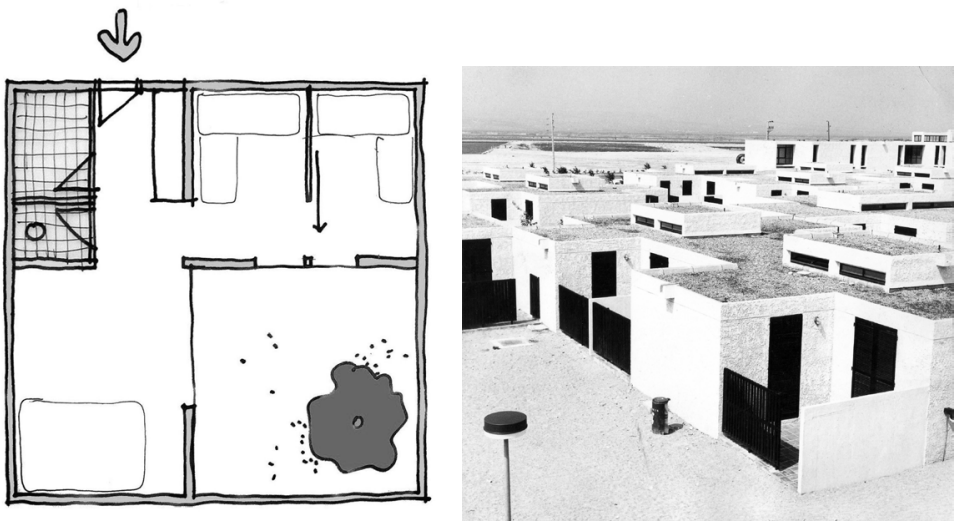
sic-Woods en los años de posguerra, que al adaptarse a las necesidades de vacaciones, resultó un tipo de grands ensembles con terrazas y logias.

La vivienda individual de la estación fue destinada a alojamiento temporal o permanente: moteles, villages de vacances, marinas y villas. El diseño de la vivienda individual ofrece al usuario una alternativa de alojamientos con libertad espacial e integración al medio ambiente y otorga al veraneante un ambiente seguro, privado y tranquilo como consecuencia de la exclusión de los automóviles.

La arquitectura de la vivienda individual de la estación retoma la propuesta de viviendas con patio inscritas en una trama horizontal elaborada por Michel Ecochard en Carrières Centrales en Casablanca. De igual manera, en la nueva estación Port Leucate-Barcarès, cada vivienda individual fue organizada alrededor de un patio y/o un pequeño jardín para responder a las necesidades mediterráneas. Este tipo de viviendas fueron llamadas villas-patio (maison à patio) (figura 16).

Las villas-patio no rebasan planta baja +2 niveles y están orientadas hacia el exterior reflejando un modo de vida estival. El patio tiene el papel de un vestíbulo al aire libre. La arquitectura es una mezcla de volúmenes y pequeños espacios exteriores protegidos del viento por muretes, celosías o cercas de madera. Los techos pueden ser inclinados o utilizados como terrazas.

Para Port Leucate-Barcarès, Candilis propuso varios tipos de acoplamientos horizontales de villas-patio: agrupadas en capa, agrupadas en banda y agrupadas en forma de puzzle. Las viviendas agrupadas, unidas por medianeras pueden formar células de dos a cuatro viviendas integradas al conjunto mediante una trama simple o en forma de puzzle. En todas las opciones de agrupación el principal elemento de articulación en la vivienda es el patio o jardín privado que contribuye a integrar la naturaleza a las viviendas (figura 17).

La disposición de las viviendas genera un sistema de áreas verdes y jardines comunitarios. El acceso de los automóviles al interior de los conjuntos fue restringido. Los automóviles fueron ubicados en núcleos de estacionamientos

Fig.18: Marina de viviendas individuales. Fuente: Aménagement touristique du littoral Languedoc-Roussillon. Techniaues et Architecture. 1969. série 31. novembre. ํㅡ 2.

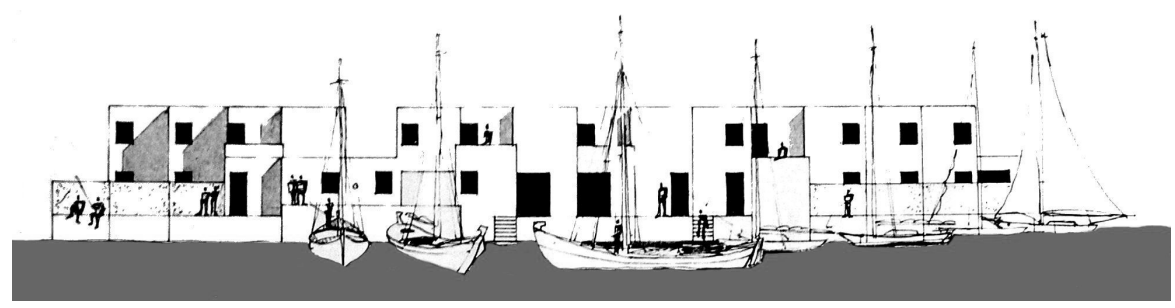


núcleos de estacionamientos. Como innovación, se propuso un pequeño tren sobre ruedas como un medio de transporte interno y para repartir a los habitantes la comida preparada en la cocina central.

La zona de habitación se subdivide en cinco unidades autónomas, cada una con 200 bungalows, 2 inmuebles colectivos y un pequeño edificio de administración. Cada unidad se conecta a la playa por un centro lineal arbolado - un corto y sencillo stem-, en el cual confluye una red de andadores peatonales. La zona de habitación se presenta como una estructura arquitectónica continua -ligero web- formada de bungalows, los cuales, previstos para cuatro camas, se desarrollan alrededor de un patio considerado como una estancia al aire libre.

Georges Candilis concedió a cada asociación responsable de las unidades del village de vacances la libertad de organizarse independientemente y practicar una animación particular. La idea rectora del proyecto se basa en tres elementos sociales base: unidad familiar, unidad vecinal y la unidad autónoma. La unidad familiar se concibe alrededor de un patio privado, la unidad vecinal en torno a plazoletas y la unidad autónoma se caracteriza por el centro lineal y los servicios generales que abastecen a todo el conjunto (figura 20).

\section{Conclusiones}

El desarrollo turístico del Languedoc-Roussillon es uno de los primeros proyectos del turismo de sol y playa en el mundo, que contó con un amplio equipo de arquitectos y urbanistas que reflexionaron respecto a las características del nuevo fenómeno y consecuentemente, sobre cuál debía ser la respuesta arquitectónica. A partir del análisis del proyecto de Port Leucate-Le Barcarès se puede afirmar que la arquitectura turística destinada para satisfacer las necesidades de un turismo masivo de sol y playa, a partir de 1960, nació en el contexto teórico del Movimiento Moderno. La arquitectura moderna encontró un espacio natural de emplazamiento cuando se enfrentó a satisfacer necesidades del turismo masivo de sol y playa, lo que motivo a los arquitectos a integrar al turismo como un elemento más en la concepción de las ciudades.

Georges Candilis, responsable del proyecto de Port Leucate-Barcarès, afrontó el proyecto desde la revisión crítica de los principios del Movimiento Moderno. La práctica lo condujo a proponer innovaciones urbanas, incorporar nuevas tipologías arquitectónicas y a reformular sus propias aportaciones teóricas para adaptarlas a las prácticas turísticas. El anteproyecto de la estación nos muestra al arquitecto Candilis en una etapa en la cual consideraba que sus conceptos teóricos, productos de muchos años de reflexión, eran susceptibles de ser utilizados fielmente como fueron concebidos para la ciudad convencional. Aunque trató de crear una arquitectura del ocio, finalmente terminó plasmando en el proyecto de la estación sus ideas preconcebidas. 
El equipamiento turístico de la estación aportó nuevas tipologías arquitectónicas como las escuelas de vela, pasillos comerciales cubiertos, equipamiento de playa (módulos de sanitarios), restaurantes de playa, pabellones comerciales y las villages de vacances, consideradas como una alternativa para el alojamiento popular. La arquitectura de las villages de vacances está basada en las megaestructuras horizontales (web) que fueron adaptadas a las necesidades del turismo, la escala urbana y a las condiciones del clima y el lugar.

Los principales principios urbanos modernos que estructuraron la nueva estación turística fueron: el orden abierto, la zonificación y el stem. Una versión en menor escala del stem es la calle comercial peatonal (rue commercial) que concentra las actividades sociales y comerciales en puntos estratégicos de la ciudad, contribuyendo a la equilibrada distribución de los servicios turísticos y el equipamiento urbano. Los pabellones comerciales o pasillos cubiertos ubicados en medio de esta calle son la muestra de una adaptación a las condiciones del clima y a las necesidades específicas del visitante, como son caminar, socializar y consumir.

Georges Candilis advierte la fuerte influencia de la civilización del ocio en la arquitectura y el urbanismo afirmando que era inevitable la incorporación del ocio en la vida cotidiana del hombre. En la estación Port Leucate-Le Barcarès el turismo se integró como una actividad más de las establecidas por la Carta de Atenas. Contrariamente a la zonificación propuesta por Le Corbusier, en la cual se separaron las diferentes funciones de una ciudad (trabajar, circular, descansar y habitar), en la estación turística la zonificación de las viviendas formó barrios distintos que presentan diversidad tipológica respecto a su densidad.

Las megaestructuras verticales propuestas en la estación por Georges Candilis tuvieron su origen en el interés de eliminar el edificio bloque aislado que conformaron los primeros grandes ensembles proponiendo formas de articulación vertical y horizontal que incrementaran la interrelación de los edificios. De igual manera, los proyectos de los edificios colectivos de la estación Port Leucate-Barcarès eliminan el tradicional edificio bloque para llegar a soluciones a $45^{\circ}$ o a largos edificios continuos rematados con volúmenes escalonados o entrelazados para facilitar la instalación de terrazas en cada vivienda.

La preocupación por el problema de la vivienda conllevó al equipo CandilisJosic-Woods a realizar estudios de diferentes tipos de acoplamientos horizontales de vivienda individual, los cuales fueron retomados para la arquitectura de la estación. Los acoplamientos de viviendas, definidos en tramas reticulares, están formados por villas-patio en las que se utiliza el patio como elemento articulador de los espacios al interior de las viviendas, al mismo tiempo que con el resto de las villas acopladas. La vivienda individual integrada al equipamiento turístico, recreativo y deportivo dentro 
de un complejo dio al turista la posibilidad de viajar a la estación de manera económica, conservar la privacidad espacial, realizar actividades lúdicas y disfrutar del contacto con el mar sin salir del complejo.

En definitiva, las soluciones arquitectónicas que el arquitecto Candilis propone para el espacio turístico buscan una respuesta un tanto más compleja que la que se había dado en el proyecto de vivienda masiva del grand ensemble. En éste, la residencia formaba parte de un puzzle en el que las cosas estaban agrupadas por usos. El conjunto estaba formado por la agrupación de piezas; de piezas mínimas. En la ciudad turística, en la que no hay grandes diferencias de usos, o mejor dicho, toda ella se orienta al servicio del ocio, el proyecto se articula entre sí como un sistema en red.

La respuesta que Candilis propone no es una respuesta unívoca, sino más bien una respuesta abierta, una línea de exploración que debía haberse seguido corrigiendo y repasando, volviendo a los proyectos y a sus opuestos para entender el valor de la relación por encima de su concreción física.

\section{Bibliografía}

CANDILIS, G.; DONY, JOSIC y WOODS, S. (1960). "Recherches pour une structure de l'habitat". L'Architecture d'Aujourd'hui. Septembre-novembre, ㄲo 91.

CANDILIS, G. (1965-1973). "Aménagement touristique du Languedoc-Roussillon. Création d'une équipe". CANGE-A-4. Dossier 236 IFA 318/1. Centre d'Archives d'architecture du XXe siècle de I'Institut français d'architecture.

(1967). "Analyse critique de la place des loisirs dans l'architecture et l'urbanisme contemporains". L'Architecture d'Aujourd'hui, avril-mai, ํo 131.

CANDILIS, G. (1977). "Bâtir la vie: un architecte témoin de son temps". Paris: Stock.

"Existe-t-il une architecture des loisirs? Un entretien avec Georges Candilis". CANGE-A-8. Dossier 236 IFA 314/14. Centre d'Archives d'Architecture du XXe siècle de l'Institut Français d'Architecture.

"Le plan de masse de l'habitat". CANGE-A-4. Dossier 236 IFA 303/7. Centre d'Archives d'Architecture du XXe siècle de l'Institut Français d'Architecture.

(1969). “Les trames directionnelles et leur utilisation". L'Architecture d'Aujourd'hui. Juin-juillet, № 144.

(1973). "Recherches sur l'architecture des loisirs". Paris: Eyrolles.

CANDILIS, G., DREYSSE y DIWI (1967). "Aménagement touristique des côtes du LanguedocRoussillon, village de vacances de l'unité de Leucate-Barcarès". L'Architecture d'Aujourd'hui. Avrilmai, no 131 .

CANDILIS, G. (1962). “'̈ la recherche d'une structure urbaine”. L'Architecture d'Aujourd'hui. Avril-mai, no 101.

(1964). "Recherches d'architecture". L'Architecture d'Aujourd'hui. Juin-juillet, no 115, p. 14-19.

(1975). "Toulouse-Le Mirail. La naissance d'une ville nouvelle". Stturgart: Kart Krämer Verlag. 
DUMAZEDIER, J. (1962). "Vers une civilisation du loisir?". Paris: Éditions du Seuil.

ÉQUIPE CANDILIS,G. (1969). "Rue Commerciale, Barcarès Leucate". L'Architecture d'Aujourd'hui. Juin-juillet, $n^{\circ} 144$.

LACAILLE, S. (2002). "L'architecture du temps libre de Georges Candilis (1963-1972): Structures pour la villégiature. Mémoire de D.E.A.: Le projet architectural et urbain: théorie et dispositifs". Paris: Écoles d'Architecture de Paris, Institut Français d'urbanisme et Ecole des Hautes Études en Sciences Sociales (Direction Yannis Tsiomis et Jean-Louis Cohen).

(1964-1965). "L'aménagement touristique des côtes du Languedoc-Roussillon" (Colloque). L'Architecture d'Aujourd'hui. , décembre-février, ํำ118, p. 24-32.

(1964). "Projet d'aménagement touristique du littoral Languedoc-Roussillon, France". L'Architecture d'Aujourd'hui Février-mars, nº 112, p. 2-4.

(1969). "Le nouveau Barcarès. Une Venise catalane". Les nouvelles littéraires, 10 avril.

(1967). "L'habitat pour le plus grand nombre". L'Architecture d'Aujourd'hui. Juin-juillet, ํo 132.

LUCAN, J. (2001). "Architecture en France (1940-2000). Histoire et théories". Paris: Éditions Le Moniteur, Collection Architextes.

MOLLE, P. "Pourquoi créer une station à Leucate-Le Barcarès?". CANGE-G. Dossier 236 IFA 317/10. Centre d'Archives d'Architecture du XXe Siècle de l'Institut Français d'Architecture.

RACINE, P. (1980). "Mission impossible?: L'aménagement touristique du littoral LanguedocRoussillon". Montpellier: Éditions du Midi Libre.

SOLOPOVA, N. (1993). "TEAM X et le groupe Candilis, Josic et Woods. Mémoire de D.E.A.: Le projet architectural et urbain: théorie et dispositifs". Paris: École d'Architecture de Paris-Belleville-La Villette (Direction Yannis Tsiomis et Jean-Louis Cohen).

Stern (1965). "Urlaub wue die Termiten". Novembre.

Techniques et Architecture (1969). "Aménagement touristique du littoral Languedoc-Roussillon". Novembre, série 31, ํำ 2, p. 31-113.

Paris-Match (1964). "Voici la Floride de demain, Le Languedoc". Août, noำ 799.

WOODS, S. (1960-1961). "Stem". Le Carré bleu, no 3.

WOODS, S. (1962). “Web”. Le Carré bleu, 끄 3 .

Izol Marez, Dra. Arquitecta Departamento de Urbanismo y Ordenación del Territorio (DUOT) Universidad Politécnica de Cataluña (UPC) Profesora Investigadora, Universidad Autónoma de Sinaloa. izol.marez@imarez.com 